\title{
Transplantation of periodontal ligament cell sheets expressing human $\beta$-defensin-3 promotes anti-inflammation in a canine model of periodontitis
}

\author{
MINWEN ZHU ${ }^{1 *}$, BO MIAO ${ }^{2 *}$, JIANHUA ZHU ${ }^{3}$, HAIYAN WANG ${ }^{1}$ and ZENGTONG ZHOU ${ }^{1}$ \\ ${ }^{1}$ Department of Oral Mucosal Diseases, Shanghai Ninth People's Hospital, Shanghai Jiao Tong University School of Medicine, \\ Shanghai 200011; ${ }^{2}$ Department of Stomatology, Aviation General Hospital of China Medical University, \\ Beijing 100012; ${ }^{3}$ Department of Periodontal Mucosa, Affiliated Stomatological Hospital \\ of Jiamusi University, Jiamusi, Heilongjiang 154002, P.R. China
}

Received December 2, 2016; Accepted July 3, 2017

DOI: $10.3892 / \mathrm{mmr} .2017 .7514$

\begin{abstract}
Periodontitis is a chronic oral inflammatory disease caused by microorganisms. Human $\beta$-defensin- 3 (HBD-3) is an endogenous antimicrobial peptide that inhibits a broad spectrum of microorganisms. Cell sheet technology has been widely applied in tissue and organ reconstructions. In the current study, it was aimed to investigate the anti-inflammatory effect of periodontal tissue engineered by HBD-3 gene-modified periodontal ligament cell (PDLC) sheets, and to identify a suitable method of promoting the regeneration of periodontal tissues. Western blot analysis and antimicrobial tests were used to confirm the expression of HBD-3. The effect of the cell sheets on anti-inflammatory activity and bone remodeling in a dog model of periodontitis was demonstrated by immunohistochemistry. The results demonstrated that the transfected PDLCs stably expressed HBD-3. Periodontal pathogens were susceptible to the antimicrobial activity of the cell sheets. In addition, the cell sheets relieved the bone resorption caused by inflammation in the in vivo model. HBD-3 may potentially be applied in the treatment of periodontitis and may function as osteogenic promoter via its anti-inflammatory effect.
\end{abstract}

Correspondence to: Dr Haiyan Wang or Professor Zengtong Zhou, Department of Oral Mucosal Diseases, Shanghai Ninth People's Hospital, Shanghai Jiao Tong University School of Medicine, 639 Zhizaoju Road, Shanghai 200011, P.R. China

E-mail: 13601957850@163.com

E-mail: zhouzengtong@hotmail.com

*Contributed equally

Key words: human $\beta$-defensin-3, anti-inflammatory, cell sheet, periodontitis, periodontal ligament cell, periodontal regeneration

\section{Introduction}

Chronic periodontitis is caused by a wide range of microorganisms and involves an inflammatory process of bacterial origin, which affects periodontal tissues and provokes the destruction of supporting tissues of the teeth. The ultimate goal of periodontal therapy is to regenerate structures diminished by the disease (1); thus, development of an antimicrobial agent and method to support regeneration of periodontal supporting tissue to protect regenerated periodontal tissue in infectious environments is required.

Defensins, a family of antimicrobial peptides, are vital contributors to host immune responses due of their ability to quickly and specifically recognize and neutralize invading microorganisms. Among the known human $\beta$-defensins (HBDs), human $\beta$-defensin-3 (HBD-3) is widely expressed in healthy individuals at readily detectable levels, and exerts strong antibacterial and immunomodulatory activities; whereas, its levels are markedly reduced in the gingival crevicular fluid of individuals with periodontitis (2). An in vitro study conducted by Lee et al (3) demonstrated that the periodontopathogens, $P$. intermedia and $T$. forsythia, have distinct susceptibilities to HBD-3. Furthermore, we previously reported that HBD-3 has important antibacterial activity and may have a role in inducing fibroblast proliferation, promoting periodontal regeneration (4). Thus, it is reasonable to hypothesize that increasing HBD-3 expression in periodontal tissue may contribute to periodontal regeneration.

Conventional treatments of periodontitis, including proper oral hygiene and scaling/root planing, aim to prevent the disease, and slow or stop its progress, however, they are usually insufficient to promote the regeneration of damaged structures (5). Cell sheet technology (CST), a novel alternative to conventional cell delivery methods, is a recently developed scaffold-free strategy that is also referred to as 'cell sheet engineering' (6). CST has been widely applied for tissue and organ reconstruction in a diverse array of tissues, including the cornea (7), esophagus (8), trachea (9), liver (10) and heart (11). Cell-based therapies have also been used to achieve periodontal regeneration, as periodontal ligament cells (PDLCs) 
have the capacity to function as osteoblasts or cementoblasts under regenerative conditions $(12,13)$.

Antimicrobial gene therapy is useful for protecting regenerated tissues/organs from infection, and reducing the need for conventional antibiotics that induce drug resistance (14). HBD-3 gene transfection was demonstrated to protect against bacterial invasion (15). Although the addition a drug is an easy for clinical use, local gene therapy has the potential to provide more sustained protein production, deliver protein in a physiological manner and allow the development of biological cellular delivery vehicles that can enhance bone repair (16). The combination of tissue engineering and antimicrobial gene therapy could potentially work hand-in-hand to make tissue repair more successful as infection is always a major risk factor during tissue restoration (14).

The aim of the present study was to investigate whether transfected PDLC sheets expressed HBD-3. Further, anti-inflammatory activity of the transfected cell sheets were evaluated in a beagle dog model of periodontitis to determine whether they provide a suitable method of promoting periodontal tissue healing and regeneration. Associated biomarkers were evaluated via immunohistochemistry (IHC).

\section{Materials and methods}

Animals. Seven female beagle dogs (12 months of age) were used in the current study. The study was approved by the Animal Experiments Ethics Committee of Shanghai Jiao Tong University School of Medicine (Shanghai, China). All of the experimental dogs had optimal periodontal health prior to the study.

Cells isolation and culture. PDLCs were isolated from the anterior teeth of each dog via enzyme digestion. The isolated PDLCs were cultured in expansion medium composed of $\alpha$-minimum essential medium ( $\alpha$-MEM) supplemented with $10 \%$ fetal bovine serum (FBS) (both from Gibco; Thermo Fisher Scientific, Inc., Waltham, MA, USA), ascorbic acid phosphate ester and $1 \%$ antibiotic-antimycotic solution (Gibco; Thermo Fisher Scientific, Inc.) at $37^{\circ} \mathrm{C}$ in a humidified atmosphere of $95 \%$ air and $5 \% \mathrm{CO}_{2}$.

Recombinant plasmid construction and transfection. HBD-3 DNA was obtained from our previous study (17). The HBD-3 gene and the green fluorescent protein (eGFP) gene were cloned into the pLV.Des3d. P/puro vector [all from Cyagen Biosciences (Guangzhou), Inc., Guangzhou, China]. E. coli Stbl3 was used as the host. For amplification, the primer sequences were as follows: attB1-Kozak-Humacalx-F 5'-GGGGACAAGTTTGTACAA AAAAGCAGGCTGCCACCATGGGCTTCCAAAAGTTCT CCC-3'; attB2-Humacalx-R, 5'-GGGGACCACTTTGTACA AGAAAGCTGGGTTTAGTTGGCATTCTGGGGCATG-3'; attB1-Kozak-PTH-F, 5'-GGGGACAAGTTTGTACAAAAA AGCAGGCTGCCACCATGATACCTGCAAAAGACATGG C-3'; attB2-PTH-R, 5'-GGGGACCACTTTGTACAAGAAGC TGGGTTCACTGGGATTTAGCTTTAGTTAATAC-3' (Sangon Biotech Co., Ltd., Shanghai, China). The polymerase chain reaction (PCR) amplification system included $5 \mathrm{X}$ primer STAR ${ }^{\mathrm{TM}}$ buffer $\left(\mathrm{Mg}^{2+}\right.$ Plus, $\left.10 \mu \mathrm{l}\right)$, dNTP mixture $(4 \mu \mathrm{l})$ (both from Takara Bio, Inc., Otsu, Japan), forward primer $1 \mu 1$, reverse primer $1 \mu \mathrm{l}$,
DNA $1 \mu \mathrm{l}$, primer STAR ${ }^{\text {TM }}$ HS DNA polymerase $(0.5 \mu l$; Takara Bio, Inc.) and $\mathrm{ddH}_{2} \mathrm{O}$. The PCR amplification conditions were as follows: $98^{\circ} \mathrm{C}$ for $3 \mathrm{~min}$, then 30 cycles of $98^{\circ} \mathrm{C}$ for $30 \mathrm{sec}, 60^{\circ} \mathrm{C}$ for $30 \mathrm{sec}$ and $72^{\circ} \mathrm{C}$ for $1 \mathrm{~min}$, and a final extension of $72^{\circ} \mathrm{C}$ for $5 \mathrm{~min}$.

The recombinant plasmid pLV.EX3d. P/puro-EF1A-Humacalx-IRES/eGFP was constructed using Gateway Technology (Invitrogen; Thermo Fisher Scientific, Inc.). The recombinant plasmid pLV.EX3d. P/puro-EF1A-PTH-IRES/eGFP was constructed without HBD-3. The constructed expression plasmids were amplified in the $E$. coli $\mathrm{Stbl} 3$ strain.

The 293T cells [American Type Culture Collection (ATCC), Manassas, VA, USA] used in transfection experiments were cultured and passaged in Dulbecco's modified Eagle's medium (DMEM) supplemented with 10\% FBS (both from Gibco, Thermo Fisher Scientific, Inc.), $100 \mathrm{U} / \mathrm{ml}$ penicillin and $100 \mu \mathrm{g} / \mathrm{ml}$ streptomycin at $37^{\circ} \mathrm{C}$ and $5 \% \mathrm{CO}_{2}$. The lentiviral vector containing HBD-3 was first transfected into $293 \mathrm{~T}$ packaging cells. Following $8 \mathrm{~h}$, the medium was replaced with fresh medium supplemented with $10 \%$ FBS. The viral particles were collected $48 \mathrm{~h}$ following plasmid transfection and were concentrated at $1,000 \mathrm{x} \mathrm{g}$ and $4^{\circ} \mathrm{C}$ for $15 \mathrm{~min}$, with filtration at $0.45 \mu \mathrm{m}$ to remove cell debris in the culture supernatant. The viral particles were then further concentrated by centrifugation at $5,000 \mathrm{x} \mathrm{g}$ for $90 \mathrm{~min}$ at room temperature. The obtained virus titer was approximately $6 \times 10^{9} \mathrm{TU} / \mathrm{ml}$ (cells transfected with HBD-3 and GFP) and $2 \times 10^{9} \mathrm{TU} / \mathrm{ml}$ (cells transfected with GFP only). The transfected PDLCs were cultured in DMEM and incubated with cells in $5 \% \mathrm{CO}_{2}$ at $37^{\circ} \mathrm{C}$. The cells were then incubated for an additional $72 \mathrm{~h}$. The transfection efficiency was calculated using a fluorescence microscope.

Western blot analysis. The GFP-HBD-3-PDLCs and GFP-PDLCs were cultured in $60 \mathrm{~mm}$ dishes until $80-90 \%$ confluency was reached. They were then collected with radioimmunoprecipitation lysis buffer (Beyotime Institute of Biotechnology, Haimen, China) and quantified using a bicinchoninic acid assay (Thermo Fisher Scientific, Inc.). Total proteins (20 $\mu \mathrm{g} /$ lane) were separated by $5-15 \%$ SDS-PAGE and transferred to PVDF membranes. Membranes were blocked with $5 \%$ nonfat milk for $1 \mathrm{~h}$ at room temperature and incubated overnight at $4{ }^{\circ} \mathrm{C}$ with the corresponding primary antibodies: Anti- $\beta$-actin (cat no. ab1801; $1: 1,000)$ and anti- $\beta$-defensin-3 antibody (cat no. ab19270; 1:1,000) (both from Abcam, Cambridge, UK). They were then incubated with a goat anti-rabbit IgG horseradish peroxidase-conjugated secondary antibody (cat no. KC-RB-035; 1:5,000; Shanghai Kangchen Biotechnology Co., Ltd., Shanghai, China) for $1 \mathrm{~h}$ at room temperature. After washing in PBST (PBS containing $0.1 \%$ Tween-20), the membranes were developed using an EZ-ECL detection kit following the manufacturer's instructions (Biological Industries Israel Beit Haemek Ltd., Beit Haemek, Israel) and were then imaged using a UVitec gel documentation system (UVitec Limited, Cambridge, UK).

Generation of PDLC sheets. GFP-HBD-3-PDLCs and GFP-PDLCs were plated into temperature-responsive culture dishes and cultured at $37^{\circ} \mathrm{C}$ in $\alpha$-MEM supplemented with $10 \% \mathrm{FBS}$, ascorbic acid phosphate ester and $1 \%$ antibiotic-antimycotic solution. When the cells reached $100 \%$ confluence, 
the dishes were transferred into an incubator set at $20^{\circ} \mathrm{C}$ to induce the formation of cell sheets. The resultant cell sheets were harvested and fixed with $10 \%$ neutral formalin solution.

ELISA. The culture supernatants were collected, and the amount of secreted HBD-3 was detected using an HBD-3 ELISA kit (cat no. JL19214; Shanghai Jiang Lai Biotechnology Co., Ltd., Shanghai, China) according to the manufacturer's instructions. Absorbance at $490 \mathrm{~nm}$ was determined with a microplate reader (BioTek ELx800; BioTek Instruments, Inc., Winooski, VT, USA). Each sample was analyzed in triplicate.

\section{Antimicrobial activity assay}

Microbial strains and culturing conditions. Actinomyces viscosus (ATCC 19246); Candida albicans (ATCC 10231); Porphyromonas gingivalis (ATCC 33277) and Streptococcus mutans (UA 159) were used to test the antimicrobial activity of the secreted HBD-3. All the strains were gifts from the Shanghai Research Institute of Stomatology. The strains were subcultured in brain heart infusion $\left(\mathrm{BHI}\right.$; $\mathrm{BBL}^{\mathrm{TM}}$ CHROMagar $^{\mathrm{TM}}$; BD Biosciences, Franklin Lakes, NJ, USA) agar plates at $37^{\circ} \mathrm{C}$ in an aerobic $\left(5 \% \mathrm{CO}_{2}\right.$ and $95 \%$ air) or anaerobic $\left(80 \% \mathrm{~N}_{2}, 10 \% \mathrm{H}_{2}\right.$ and $\left.10 \% \mathrm{CO}_{2}\right)$ system for $24 \mathrm{~h}$.

In vitro antimicrobial activity of $H B D-3$ protein. Cell-free culture supernatants from the transfected PDLC sheets were collected $48 \mathrm{~h}$ after transfection and were serially diluted. Subsequently, $100 \mu \mathrm{l}$ suspension of the four microbial strains $\left(10^{6} \mathrm{CFU} / \mathrm{ml}\right)$ were inoculated in BHI broth supplemented with $100 \mu \mathrm{l}$ diluted supernatants at $37^{\circ} \mathrm{C}$ for $48 \mathrm{~h}$. Microbial growth was assessed by measuring the optical density (OD) at $590 \mathrm{~nm}$ with a microplate reader. All experiments were performed in triplicate. Medium alone and untreated cells were used in the control groups.

The four microbial strains in cell-free culture supernatants from $35 \mathrm{~mm}$ dish were diluted $10^{6}$, and $100 \mu \mathrm{l}$ of the solution were spread on BHI agar plates at $37^{\circ} \mathrm{C}$. The number of colony-forming units (CFU) was counted after $48 \mathrm{~h}$ incubation. All experiments were performed in triplicate. Medium alone and untreated cells were used in the control groups.

Experimental periodontitis model. The experimental dogs were fed a high sugar, high starch diet (daily diet of dog food mixed with $100 \mathrm{~g}$ sugar and $20 \mathrm{~g}$ mashed potatoes). In addition, elastic ligatures were subgingivally placed around the necks of all 3rd and 4th premolars (P3, P4) and all 1st molars (M1) to induce experimental periodontitis. The development and progression of periodontitis was monitored for 1 month.

Transplantation of PDLC sheets. Following intraperitoneal anesthesia and elevation of the buccal mucoperiosteal flap, the alveolar bone of the dogs was removed to create experimental periodontal bone defects. The defect was $5 \mathrm{~mm}$ in width, $45 \mathrm{~mm}$ in length, and $3 \mathrm{~mm}$ in depth, prepared from P3 to mesial root of M1.

The four sites of experimental periodontitis in each dog were randomly assigned into the following three experimental groups: Test group, transplanted with GFP-HBD-3-PDLC sheets (Fig. 1); control group, transplanted with GFP-PDLC sheets (Fig. 1); and untreated group, which received no treatment. The grouping results were as follows: 28 sites were involved in the study and there were 9,12 and 7 sites assigned into the test group, the control group and the untreated group, respectively. To ensure that a uniform thickness was maintained between the different constructs, the same layers of cell sheets were used, and all procedures were completed by the same person. Following the surgery, all dogs used in this experiment received $4 \times 10^{6}$ units amoxicillin intramuscularly daily for 7 days.

Tissue preparation and IHC. Inflammation during the healing period was observed 2 weeks following transplantation in the diseased regions of the untreated and control groups. Assessments were made by eye using clinical observations including, gingival swelling, periodontal overflow pus and loose teeth. At 8 weeks after the transplantation with cell sheets, the periodontitis model dogs were sacrificed and the alveolar bones of experimental sites were harvested. After excision, fixation in $4 \%$ paraformaldehyde for $24 \mathrm{~h}$ at room temperature and demineralization in $10 \%$ EDTA for 10 weeks at room temperature, buccal-lingual histologic specimens from P3, P4 and M1, each containing a tooth and its associated periodontium, were embedded in paraffin wax. Serial tissue sections $(3-\mu$ m-thick) from the paraffin blocks were stained with hematoxylin and eosin for $5 \mathrm{~min}$, respectively, at room temperature for cell visualization.

Following deparaffinization, antigen retrieval was performed with $1 \mathrm{mM}$ Tris-EDTA $(\mathrm{pH} 8.0)$ in a $100^{\circ} \mathrm{C}$ water bath for 20 min and endogenous peroxidase activity was blocked with $3 \%$ hydrogen peroxide for $10 \mathrm{~min}$ at room temperature. All of the sections were then incubated with primary antibodies against GFP (cat no. ab183735; 1:200), tumor necrosis factor- $\alpha$ (TNF- $\alpha$; cat no. ab199013; 1:200), interleukin-1 $\beta$ (IL-1 $\beta$; cat no. ab34837; 1:200) and osteocalcin (OCN; cat no. ab13418; $10 \mu \mathrm{g} / \mathrm{ml}$ ) (all from Abcam) for $1 \mathrm{~h}$ at room temperature. An EnVision ${ }^{\mathrm{TM}}$ Detection Systems kit (Peroxidase/DAB; cat no. K5007; Dako; Agilent Technologies, Inc., Santa Clara, CA, USA) was applied until the desired staining intensity was achieved, which was observed under a light microscope (Axio Scope A1; Carl Zeiss AG, Oberkochen, Germany). To ensure that pathological diagnosis was standardized, the cellular localization patterns, intensities, and numbers of cells with positive staining were assessed by two experienced oral pathologists in a double-blind manner. A consensus decision was reached in cases with discrepancies. Staining intensity was defined as 0 , 1, 2 and 3 for no staining, light yellow staining, yellow-brown staining and brown staining, respectively. Expression levels were semi-quantitatively evaluated by multiplying the mean percentage of positive cells by the intensity score. The mean percentage of positive cells was determined in 4 random fields (magnification, $\mathrm{x} 400$ ).

Statistical analysis. SPSS 19.0 software package (IBM Corp., Armonk, NY, USA) was used for all statistical analyses. Significant differences were calculated using one-way analysis of variance followed by the least significant difference or Tamhane test. The ELISA and antimicrobial activity assay data are expressed as the mean \pm standard error. The IHC are expressed as the mean \pm standard deviation. $\mathrm{P}<0.05$ was considered to indicate a statistically significant difference. 


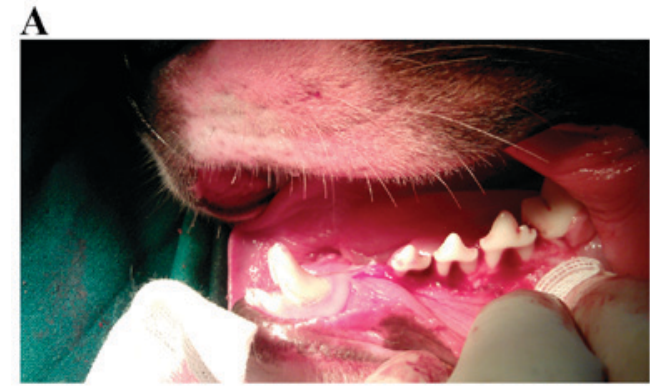

B

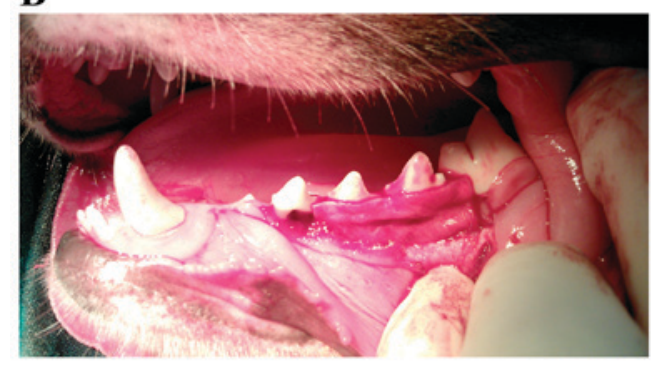

Figure 1. Sites of experimental periodontitis were transplanted with the cell sheets. (A) Transplanted defect regions were formed on the buccal side of the root furcation of 3rd premolars, 4th premolars and 1st molars. (B) GFP-HBD-3-PDLC sheets or GFP-PDLC sheets were applied to the experimental defects. GFP, green fluorescent proteins; HBD-3, human $\beta$-defensin-3; PDLC, periodontal ligament cell.
A

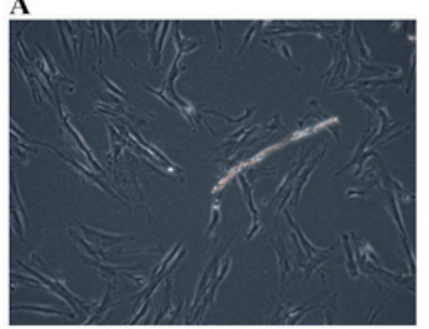

C

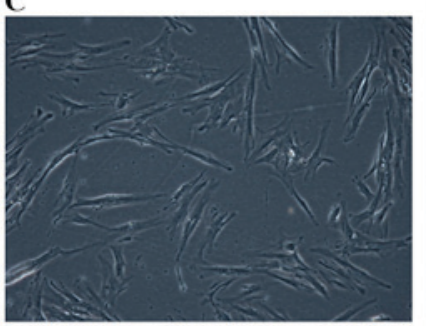

B

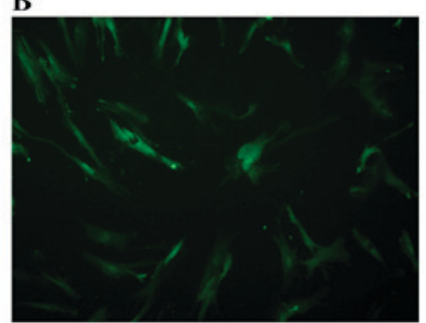

D

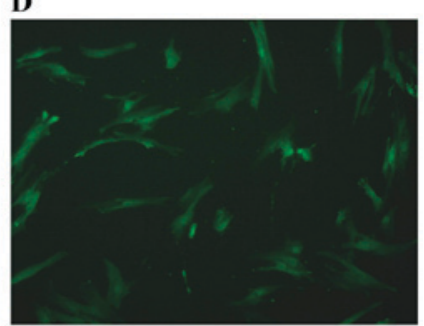

Figure 2. PDLCs transfected with a lentiviral vector containing the HBD-3 and GFP genes. (A) Optical microscopy and (B) fluorescence microscopy images of PDLCs transfected with the GFP-HBD-3 lentivirus. (C) Optica microscopy and (D) fluorescence microscopy images of PDLCs transfected with the GFP lentivirus only. GFP, green fluorescent proteins; HBD-3, human $\beta$-defensin-3; PDLCs, periodontal ligament cells.

\section{Results}

Transfection efficiency. Transfection efficiency was calculated by counting the cells that fluoresced green. The results demonstrated that the rate of PDLC transfection with the GFP + HBD-3 vector was $78.98 \%$ (Fig. 2A and B). The rate of PDLCs transfection with the GFP only vector was $74.96 \%$ (Fig. 2C and D).

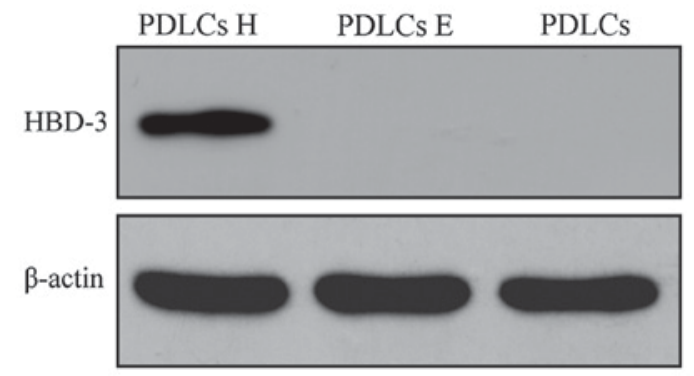

Figure 3. HBD-3 protein expression detected by western blotting. PDLCs $\mathrm{H}$ represent the PDLCs transfected with the HBD-3 and GFP genes; PDLCs E represent the PDLCs transfected with the GFP gene only. GFP, green fluorescent proteins; HBD-3, human $\beta$-defensin-3; PDLCs, periodontal ligament cells.

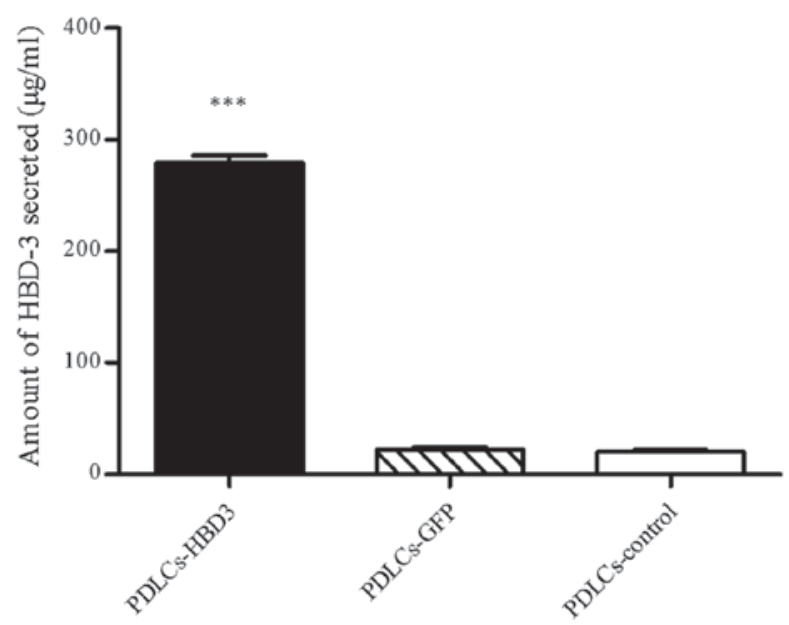

Figure 4. Amount of HBD-3 secreted by PDLCs, as determined by ELISA Statistical comparisons were performed using one-way analysis of variance. Values are expressed as the mean \pm standard error. ${ }^{* * *} \mathrm{P}<0.001$ vs. control cells. GFP, green fluorescent proteins; HBD-3, human $\beta$-defensin-3; PDLCs, periodontal ligament cells.

Western blot analysis. To examine the protein expression level of HBD-3 in the transfected PDLCs, western blot analysis was performed. Distinct positive bands were observed corresponding to the PDLCs transfected with the HBD-3 + GFP vector. However, no positive bands were observed in the untransfected groups and GFP only vector (Fig. 3).

ELISA. ELISA was performed to quantify the levels of HBD-3 in the PDLC sheets transfected with the HBD-3 and GFP genes. The untreated cell sheets were used as a control. The results showed that the concentrations of secreted HBD-3 were $279.22 \pm 2.17 \mu \mathrm{g} / \mathrm{ml}$ in the supernatants of PDLC sheets transfected with HBD-3 (Fig. 4), which was significantly higher than that of the corresponding cell sheets transfected with the control vector and that of the untreated control group $(\mathrm{P}<0.001)$.

In vitro antimicrobial activity. As presented in Fig. 5A, Actinomyces viscosus, Candida albicans, Porphyromonas gingivalis and Streptococcus mutans were cultured with supernatants from PDLC sheets transfected with the HBD-3 gene 
A
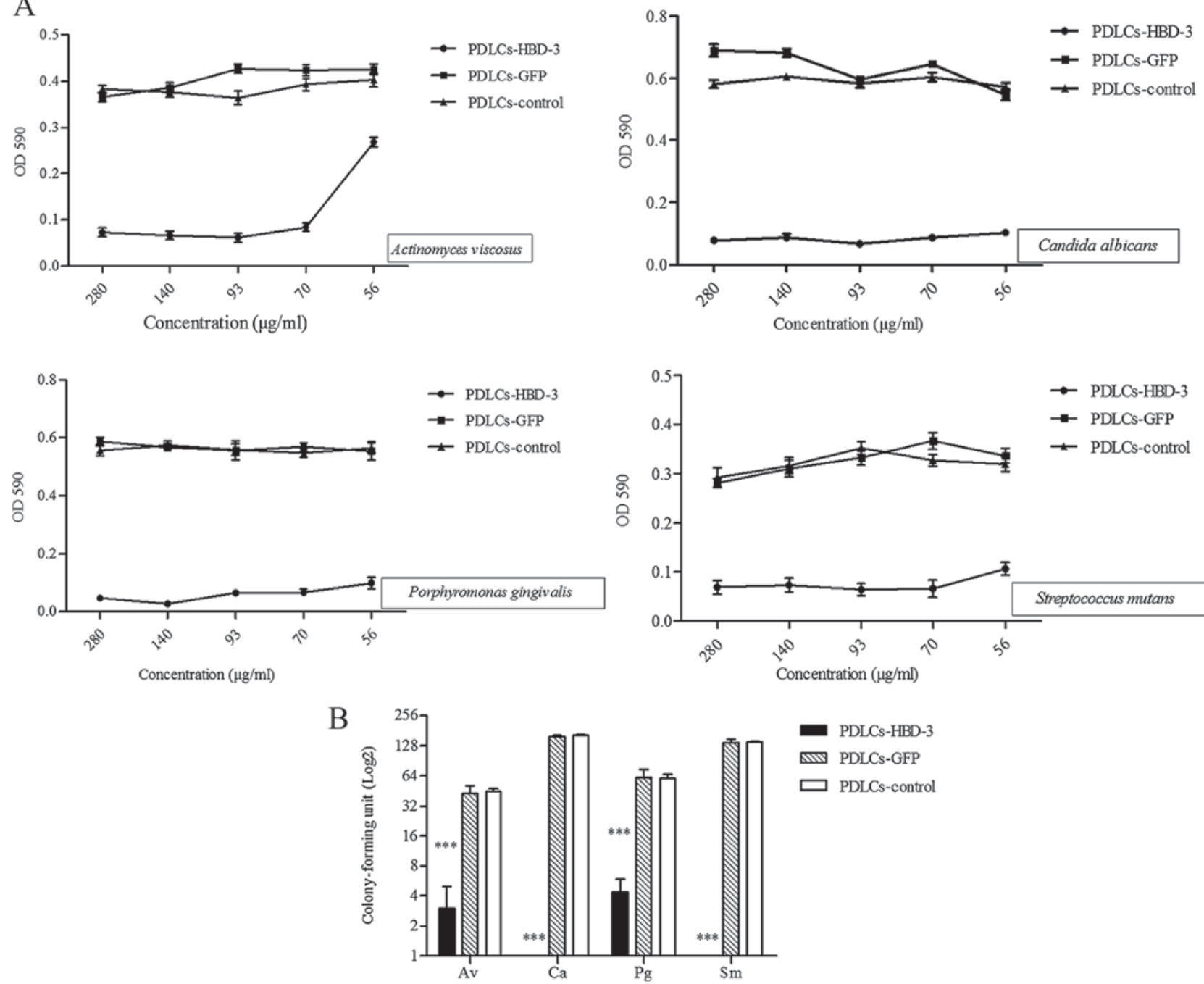

Figure 5. Antimicrobial analysis of PDLC sheets transfected with HBD-3. (A) Microbial growth was assessed by measuring OD at 590 nm with a microplate reader. (B) Colony-forming units assay. Statistical comparisons were performed using one-way analysis of variance. Values are expressed as the mean \pm standard error. ${ }^{* * *} \mathrm{P}<0.001$ vs. GFP only and control group. OD optical density; GFP, green fluorescent proteins; HBD-3, human $\beta$-defensin-3; PDLCs, periodontal ligament cells; Av, Actinomyces viscosus; Ca, Candida albicans; Pg, Porphyromonas gingivalis; Sm, Streptococcus mutans.

expression vector at various dilutions, and exhibited lower OD values compared with those cultured with supernatant from control vector and untransfected cells.

A CFU assay was also performed to evaluate the effects of HBD-3 on the antimicrobial activity of the supernatant of cell sheets against the microbial strains. Supernatant from the PDLC sheets transfected with the HBD-3 vector demonstrated significantly lower colony counts compared with those of the control cell sheets $(\mathrm{P}<0.001$; Fig. 5B).

Clinical observations of animals. During the course of the experiment, no apparent adverse reactions, such as severe infection and suppuration, were detected around the periodontal tissue. Although root exposure was observed in most dogs of all the groups, and gingival recession was marginally more severe in the control group and the untreated group than in the test group (Fig. 6).

Histopathological analysis. Different degrees of epithelium attachment to the cementum-enamel junction were observed among the three experimental groups. Less inflammatory infiltration was observed in the epithelium, and gingival and periodontal ligament fibers were arranged in a more orderly and neat fashion in the test group compared with those in the untreated group. In the test group (Fig. 7A) and the control group (Fig. 7B), a number of osteoblasts and osteocytes, as well as newly formed alveolar bone with trabeculae, were observed. By contrast, osteoclasts, visible erosion of gingival epithelia and obvious absorption of the alveolar crest were observed in the untreated group (Fig. 7C).

Immunohistochemical findings. In the test and the control group, a vast amount of GFP-positive cells could be observed in the gingiva of the experimental area (Fig. 8A) and scattered small amounts in the alveolar bone (Fig. 8B).

TNF- $\alpha$-positive cells, predominantly macrophages, were distributed throughout the gingival epithelium, periodontal membrane matrix and osteoclasts. In the test group, only weak positive staining was observed (Fig. 9A), whereas in the control group (Fig. 9B.) and the untreated group (Fig. 9C.), 

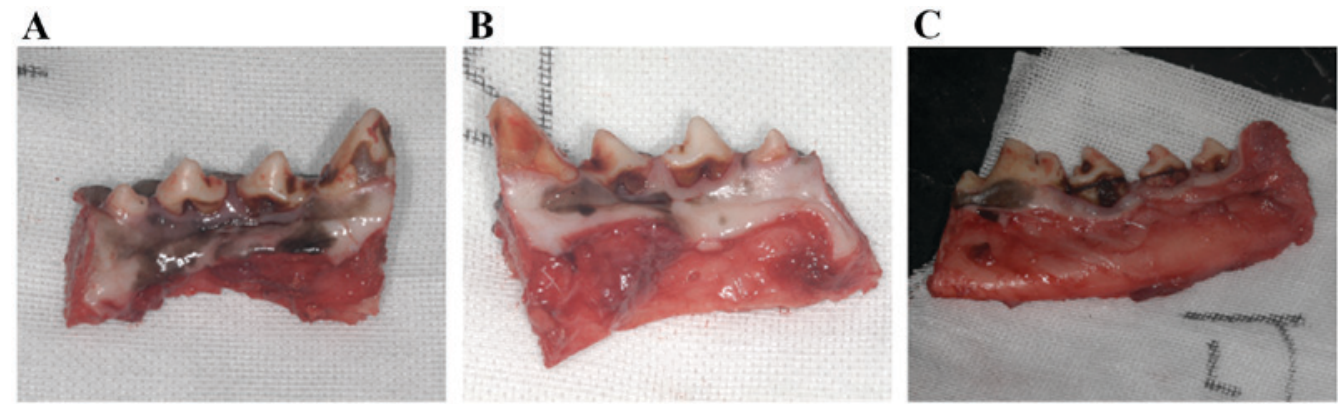

Figure 6. Root exposure was observed in all the experimental groups. Compared to that in (A) the test group, gingival recession was more severe in (B) the control group and $(\mathrm{C})$ the untreated group.

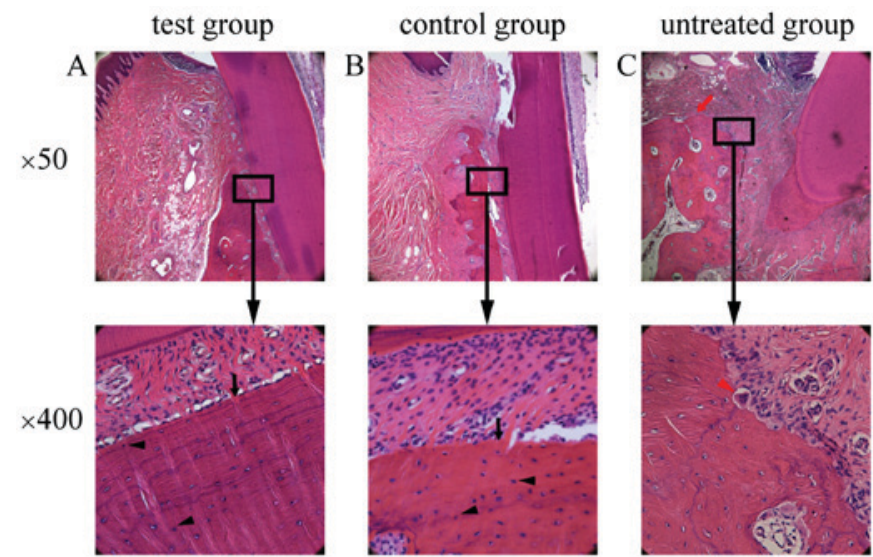

Figure 7. Histology of sections from the defect areas. Newly formed bone (black arrow) and osteoblasts (black arrow head) were observed in the defect of (A) the test group and (B) the control group. Osteoclasts (red arrow head) and obvious absorption of the alveolar crest (red arrow) were observed in the untreated group (C). Lower images are magnified view of the boxed areas.

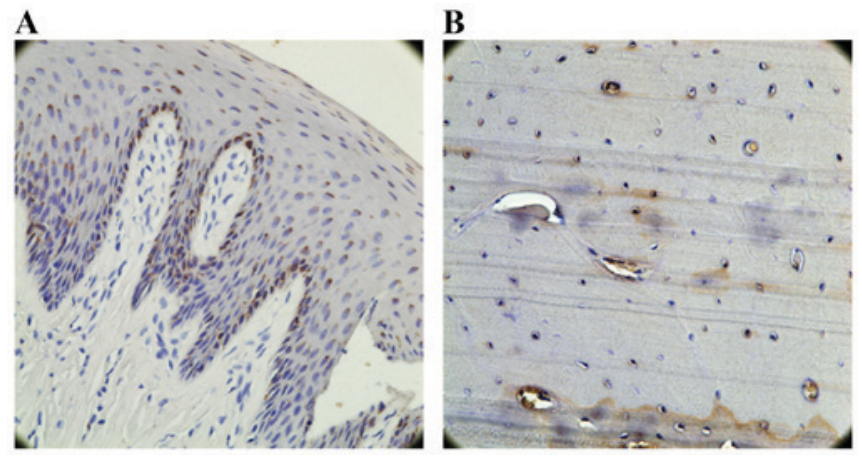

Figure 8. GFP-positive cells within (A) the periodontal ligament tissues and (B) the alveolar bone of the HBD-3 + GFP group (original magnification, $\mathrm{x} 400)$. GFP, green fluorescent protein.

TNF- $\alpha$ expression was significantly elevated compared with the test group $(\mathrm{P}<0.05$; Fig. 10). There was no significant difference between the untreated group and the control group ( $\mathrm{P}>0.05$; Fig. 10).

Similar to the TNF- $\alpha$-positive staining patterns described above, IL-1 $\beta$-positive cells were primarily observed in the gingival epithelium, periodontal membrane matrix, osteoblasts and osteoclasts (Fig. 11). In the test group, only weak IL-1 $\beta$ expression was observed, and this expression was

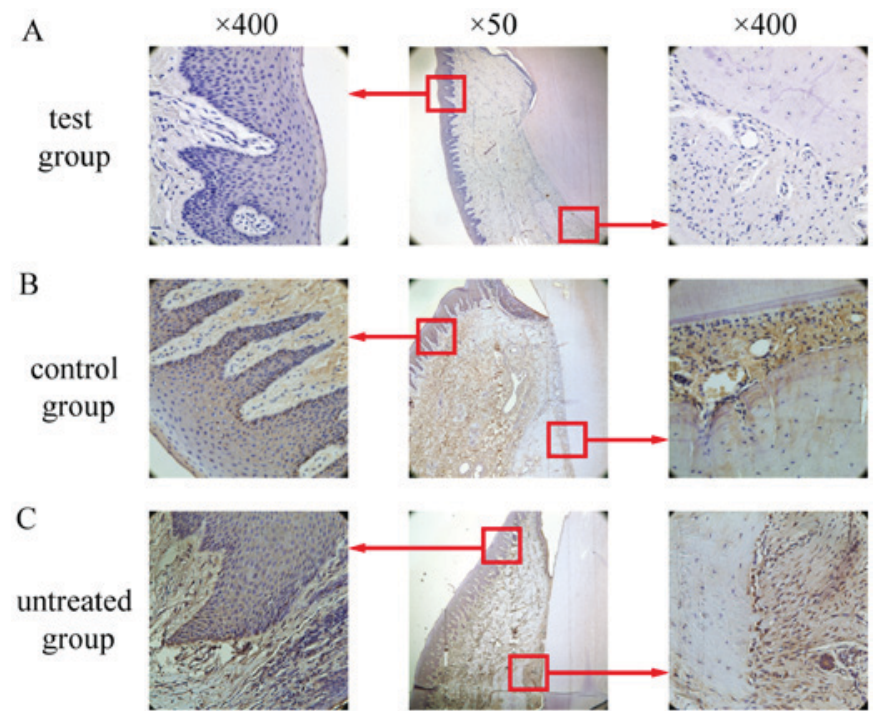

Figure 9. Tumor necrosis factor- $\alpha$ expression determined by immunohistochemistry in the experimental groups. (A) Low expression in the test group. (B) High expression in the control group. (C) High expression in the untreated group. Boxed areas are also presented at x400 magnification.

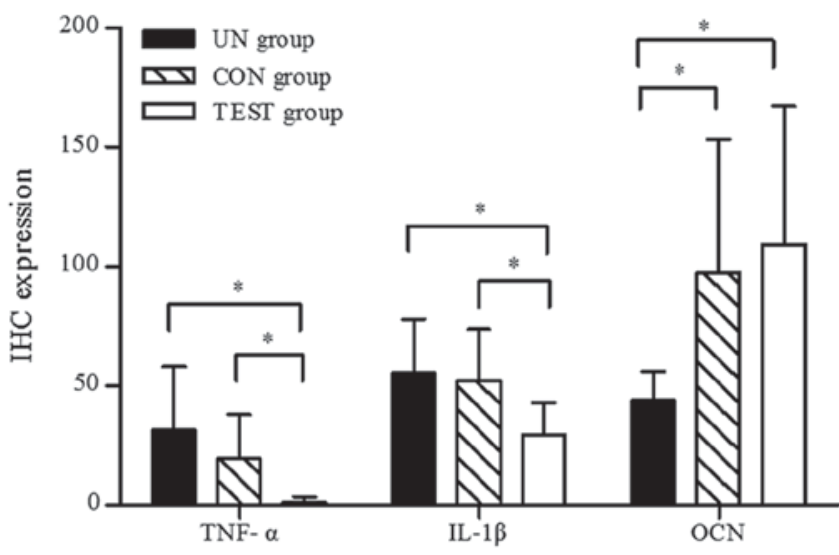

Figure 10. Comparison of the associated biomarkers detected using IHC among experimental groups analyzed by one-way analysis of variance. Values are expressed as the mean \pm standard deviation. "P<0.05. IHC, immunohistochemistry; UN, untreated; CON, control. TNF- $\alpha$, tumor necrosis factor- $\alpha$; IL-1 $\beta$, interleukin- $1 \beta$; OCN, osteocalcin.

significantly lower than that in the control group and the untreated group (Fig. 10; $\mathrm{P}<0.05$ ). No differences in IL-1 $\beta$ 
A

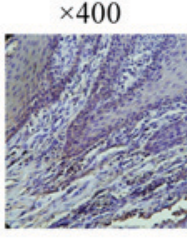

B

control group

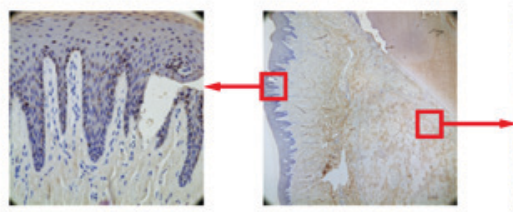

$\mathrm{C}$

untreated group

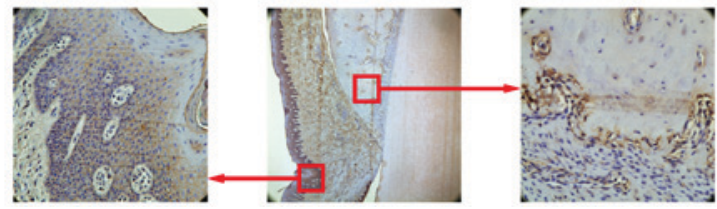

Figure 11. Expression of interleukin-1 $\beta$ in the experimental groups. (A) Low expression in the test group. (B) High expression in the control group. (C) High expression in the untreated group. Boxed areas are also presented at $\times 400$ magnification.
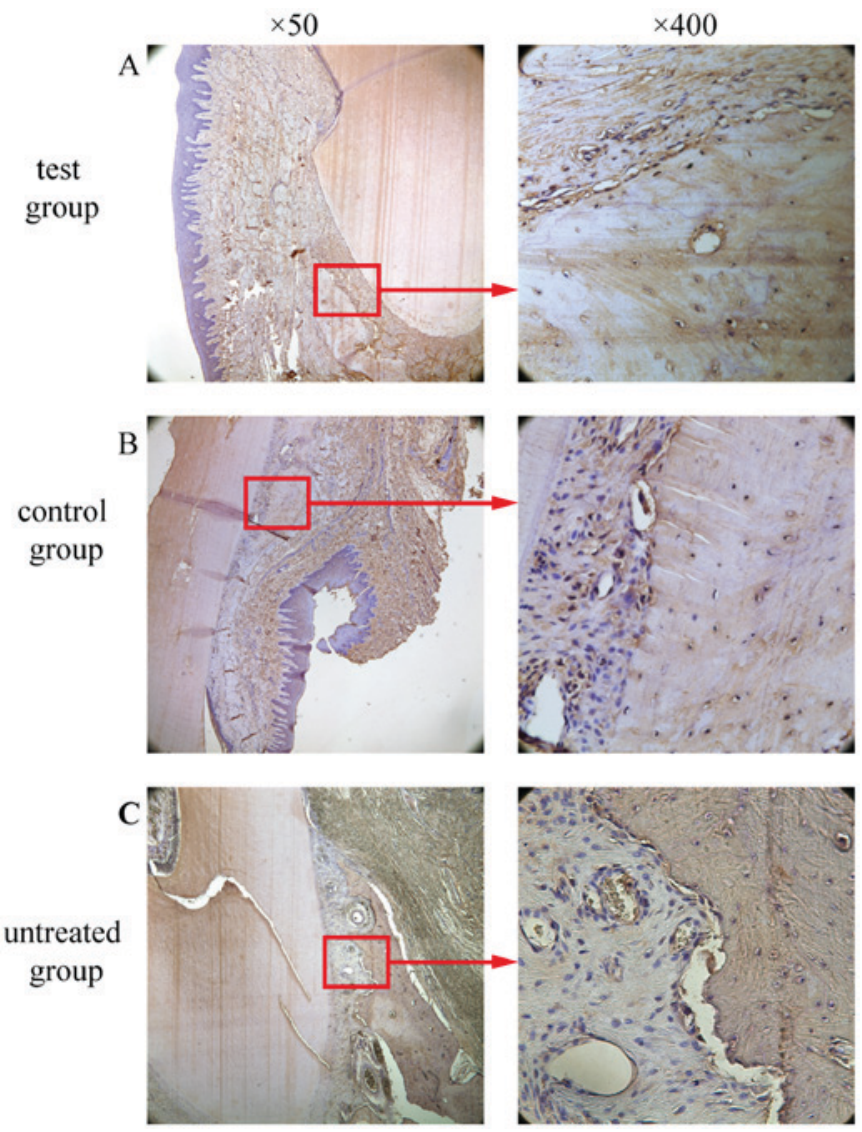

Figure 12. Expression of OCN in the experimental groups. (A) High expression of osteocytes and osteoblasts in the test group. (B) High expression of osteocytes and osteoblasts in the control group. (C) Low expression in the untreated group. Boxed areas are also presented at $\mathrm{x} 400$ magnification. OCN, osteocalcin.

expression between the untreated group and the control group were detected ( $\mathrm{P}>0.05$; Fig. 10).
OCN-positive cell counting. A marker for bone tissue, $\mathrm{OCN}$ immunoreactivity was observed in cells embedded in the alveolar bone and surrounding tissues. In the test group (Fig. 12A) and the control group (Fig. 12B), the bone and the extracellular matrix of the periodontal ligament showed moderate to strong OCN-positive staining. In the untreated group, the extracellular matrix and some cells in the alveolar bone exhibited weak staining for OCN (Fig. 12C). OCN staining was significantly higher in the test group compared to the untreated group $(\mathrm{P}<0.05$; Fig. 10). There was no significant difference in $\mathrm{OCN}$ staining between the test group and the control group ( $\mathrm{P}>0.05$; Fig. 10).

\section{Discussion}

Periodontal diseases are highly prevalent and affect up to $90 \%$ of the population worldwide (18). Pathogenic bacteria are widely recognized to be a major cause of periodontal tissue destruction, and the goals of periodontal treatments are to support good oral hygiene and regenerate tissue integrity, which may have been damaged by the inflammatory process (19).

Currently, periodontal regeneration is shifting towards celland gene-based therapies (20). Certain studies have reported that PDLCs have the capacity to function as osteoblasts or cementoblasts under regenerative conditions, suggesting that they are candidates for regeneration applications $(12,21)$. The crucial steps of gene therapy include the efficient transfer and appropriate expression of the target gene. In the present study, the efficiency of HBD-3 transfection into the PDLCs was assessed. The HBD-3 protein levels in the transfected PDLCs were sustained and were significantly higher than those of the control group.

Furthermore, the antimicrobial activity of the PDLC sheets transfected with a lentivirus containing the HBD-3 gene was determined. In the present study, periodontal pathogens, Actinomyces viscosus, Porphyromonas gingivalis, and Candida albicans $(22,23)$, were demonstrated to be susceptible to the culture supernatant from cells transfected with the HBD-3 vector compared with those in the control vector group and the untreated group. The results indicated that the transfected cell sheets expressed the HBD-3 and has the capacity to inhibit broad-spectrum microbial activity in vitro, which is consistent with the results of our previous study (24) and with other reports $(25,26)$. Notably, the caries-causing bacteria Streptococcus mutans (27) was also susceptible to HBD-3 in the experimental group.

Previous studies have demonstrated that HBD is susceptible to degradation and inactivation by both host and bacterial proteases. It has also been reported that inflamed gingival tissues expressed lower levels of HBD-3 mRNA than healthy tissues $(28,29)$. Brancatisano et al (2) detected HBD-3 using ELISA and observed that the levels were inversely correlated with the severity of the disease and with the degree of colonization by combinations of bacterial species with elevated periodontopathogenic potential. Based on these findings, it is reasonable to hypothesize that aggressive inflammation and tissue destruction occur when the HBD-3 peptide cannot counteract the microbial activity. Thus, the transfection of PDLC sheets with HBD-3 may have favorable effects on antimicrobial activity by complementing the low levels of 
HBD-3 in aggressive periodontitis and other oral microbial diseases.

PDLC sheets have demonstrated osteogenic potential in the reconstruction of periodontal tissues destroyed by chronic inflammation $(30,31)$. In the present study, multi-layer PDLC sheets were produced using autologous cells collected from beagle dogs. Following an 8 -week experimental period, the alveolar bone from the dogs in the test group and the control group was examined. The bone exhibited newly formed tissue, and no teeth were loosened or lost. By contrast, in the untreated group, root exposure, gingival recession and tooth loss were observed.

Clinical transplantation is difficult to perform and poses a risk of infection. It has been reported that an inflammatory microenvironment reduces the osteogenic differentiation potential of PDLCs $(20,32)$. In addition, inflammation of the periodontium leads to destruction of the underlying ligament and alveolar bone and an imbalance in bone remodeling that favors resorption. Few studies have examined the role of HBD-3 in periodontal regeneration. In the present in vivo study, PDLCs were transfected with HBD-3 and PDLC sheets were created for use in an experimental periodontitis model. As mediators of inflammatory responses, TNF- $\alpha$ and IL-1 $\beta$ are two pro-inflammatory cytokines involved in the process of bone resorption under pathological conditions. The protein expression levels of these two cytokines were detected in the current study using IHC. The results demonstrated that TNF- $\alpha$ and IL-1 $\beta$ expression was significantly lower in the test group (PDLC sheets expressing HBD-3) compared with the two control groups. These results demonstrate the anti-inflammatory effects of HBD-3 in the healing of periodontal disease. Following $\sim 2$ weeks after transplantation, moderate inflammation was observed in diseased regions in the untreated group and the control group, although intense inflammation was not observed in any of the experimental groups by the end of the study. The reason for this finding may be associated with the function of HBD-3 in autologous periodontal tissues; based on the above findings, HBD-3 has favorable anti-inflammatory activity in the context of oral infectious disease, particularly during the early stages of periodontitis.

Immunolocalization of OCN, an osteoblast-specific marker, produced complementary results to the aforementioned clinical observations and histopathological findings. There was significantly greater positive OCN staining in the test group compared with the untreated group. Stronger positive staining for OCN was observed in the periodontal ligament and alveolar bone in the test group than in the control group, although with no significant differences between the groups. These data suggest that HBD-3 can be used to neutralize toxins, and promote osteogenesis via its anti-inflammatory effects within the inflammatory microenvironment.

In conclusion, application of the lentiviral vector containing HBD-3 has great potential as an efficient gene therapy strategy to exert for anti-inflammatory activity in periodontitis. Bone remodeling is a complex process that involves both bone formation and bone resorption. Sheets of PDLC transfected with the gene encoding HBD-3 may decelerate bone resorption caused by inflammation, and this approach may offer a novel and safe method for enhancing periodontal regeneration during the bone remodeling process.

\section{Acknowledgements}

Dr Chunye Zhang and Dr Yuhua Hu (Department of Oral Pathology, Shanghai Ninth People's Hospital, Shanghai Jiao Tong University School of Medicine, Shanghai, China) are thanked for their technical support. This study was supported by the National Natural Science Foundation of China (grant no. 81271157).

\section{References}

1. Wang HL and Cooke J: Periodontal regeneration techniques for treatment of periodontal diseases. Dent Clin North Am 49: 637-659, vii, 2005.

2. Brancatisano FL, Maisetta G, Barsotti F, Esin S, Miceli M, Gabriele M, Giuca MR, Campa M and Batoni G: Reduced human beta defensin 3 in individuals with periodontal disease. J Dent Res 90: 241-245, 2011.

3. Lee SH, Jun HK, Lee HR, Chung CP and Choi BK: Antibacterial and lipopolysaccharide (LPS)-neutralising activity of human cationic antimicrobial peptides against periodontopathogens. Int J Antimicrob Agents 35: 138-145, 2010.

4. Wang $\mathrm{H}$, Watanabe $\mathrm{H}$, Ogita $\mathrm{M}$, Ichinose $\mathrm{S}$ and Izumi Y: Effect of human beta-defensin-3 on the proliferation of fibroblasts on periodontally involved root surfaces. Peptides 32: 888-894, 2011.

5. Meng X, Li M, Wang X, Wang Y and Ma D: Both CD133+ and CD133- subpopulations of A549 and H446 cells contain cancer-initiating cells. Cancer Sci 100: 1040-1046, 2009.

6. Liu WH, Qian NS, Li R and Dou KF: Replacing Hoechst33342 with Rhodamine123 in isolation of cancer stem-like cells from the MHCC97 cell line. Toxicol In Vitro 24: 538-545, 2010.

7. Ferrand A, Sandrin MS, Shulkes A and Baldwin GS: Expression of gastrin precursors by CD133-positive colorectal cancer cells is crucial for tumour growth. Biochim Biophys Acta 1793: 477-488, 2009.

8. Bexell D, Gunnarsson S, Siesjö P, Bengzon J and Darabi A: $\mathrm{CD} 133^{+}$and nestin ${ }^{+}$tumor-initiating cells dominate in N29 and N32 experimental gliomas. Int J Cancer 125: 15-22, 2009.

9. Barcelos LS, Duplaa C, Kränkel N, Graiani G, Invernici G, Katare R, Siragusa M, Meloni M, Campesi I, Monica M, et al: Human $\mathrm{CD}_{133^{+}}$progenitor cells promote the healing of diabetic ischemic ulcers by paracrine stimulation of angiogenesis and activation of Wnt signaling. Circ Res 104: 1095-1102, 2009.

10. Boivin D, Labbé D, Fontaine N, Lamy S, Beaulieu E, Gingras D and Béliveau R: The stem cell marker CD133 (prominin-1) is phosphorylated on cytoplasmic tyrosine- 828 and tyrosine- 852 by Src and Fyn tyrosine kinases. Biochemistry 48: 3998-4007, 2009.

11. Zhou J, Zhu B, DU HY, Sun TS, Zhang CH and Yang LG: Detection and analysis of CD271, CD133 and CD34 expressions in bone marrow cells by flow cytometry with three color fluorescence labelling. Zhongguo Shi Yan Xue Ye Xue Za Zhi 17: 133-136, 2009 (In Chinese).

12. Polimeni G, Xiropaidis AV and Wikesjö UM: Biology and principles of periodontal wound healing/regeneration. Periodontol 2000 41: 30-47, 2006.

13. Shi M, Ishikawa M, Kamei N, Nakasa T, Adachi N, Deie M, Asahara T and Ochi M: Acceleration of skeletal muscle regeneration in a rat skeletal muscle injury model by local injection of human peripheral blood-derived CD133-positive cells. Stem Cells 27: 949-960, 2009.

14. Huang GT, Zhang HB, Kim D, Liu L and Ganz T: A model for antimicrobial gene therapy: Demonstration of human beta-defensin 2 antimicrobial activities in vivo. Hum Gene Ther 13: 2017-2025, 2002.

15. Sawamura D, Goto M, Shibaki A, Akiyama M, McMillan JR, Abiko Y and Shimizu H: Beta defensin-3 engineered epidermis shows highly protective effect for bacterial infection. Gene Ther 12: 857-861, 2005.

16. Zhu L, Chuanchang D, Wei L, Yilin C and Jiasheng D: Enhanced healing of goat femur-defect using BMP7 gene-modified BMSCs and load-bearing tissue-engineered bone. J Orthop Res 28: 412-418, 2010.

17. Zhu M, Miao B, Zhu J, Wang H and Zhou Z: Expression and antimicrobial character of cells transfected with human $\beta$-defensin-3 against periodontitis-associated microbiota in vitro. Mol Med Rep 16: 2455-2460, 2017. 
18. Pihlstrom BL, Michalowicz BS and Johnson NW: Periodontal diseases. Lancet 366: 1809-1820, 2005.

19. Wang HL, Greenwell H, Fiorellini J, Giannobile W, Offenbacher S, Salkin L, Townsend C, Sheridan P and Genco RJ Research, Science and Therapy Committee: Periodontal regeneration. J Periodontol 76: 1601-1622, 2005.

20. Rios HF, Lin Z, Oh B, Park CH and Giannobile WV: Cell- and gene-based therapeutic strategies for periodontal regenerative medicine. J Periodontol 82: 1223-1237, 2011.

21. Park JY, Jeon SH and Choung PH: Efficacy of periodontal stem cell transplantation in the treatment of advanced periodontitis. Cell Transplant 20: 271-285, 2011.

22. Grant MM, Kolamunne RT, Lock FE, Matthews JB, Chapple IL and Griffiths HR: Oxygen tension modulates the cytokine response of oral epithelium to periodontal bacteria. J Clin Periodontol 37: 1039-1048, 2010.

23. Järvensivu A, Hietanen J, Rautemaa R, Sorsa T and Richardson M: Candida yeasts in chronic periodontitis tissues and subgingival microbial biofilms in vivo. Oral Dis 10: 106-112, 2004.

24. Mimeault M, Johansson SL, Henichart JP, Depreux P and Batra SK: Cytotoxic effects induced by docetaxel, gefitinib, and cyclopamine on side population and nonside population cell fractions from human invasive prostate cancer cells. Mol Cancer Ther 9: 617-630, 2010

25. Ouhara K, Komatsuzawa H, Yamada S, Shiba H, Fujiwara T, Ohara M, Sayama K, Hashimoto K, Kurihara H and Sugai M: Susceptibilities of periodontopathogenic and cariogenic bacteria to antibacterial peptides, \{beta\}-defensins and LL37, produced by human epithelial cells. J Antimicrob Chemother 55: 888-896, 2005.

26. Xia Z, Zhang C, Zeng Y, Wang T and Ai G: Transplantation of BMSCs expressing hVEGF165/hBD3 promotes wound healing in rats with combined radiation-wound injury. Int Wound J 11: 293-303, 2014
27. Costalonga M and Herzberg MC: The oral microbiome and the immunobiology of periodontal disease and caries. Immunol Lett 162: 22-38, 2014.

28. Bissell J, Joly S, Johnson GK, Organ CC, Dawson D, McCray PB $\mathrm{Jr}$ and Guthmiller JM: Expression of beta-defensins in gingival health and in periodontal disease. J Oral Pathol Med 33: 278-285, 2004.

29. Hosokawa I, Hosokawa Y, Komatsuzawa H, Goncalves RB Karimbux N, Napimoga MH, Seki M, Ouhara K, Sugai M, Taubman MA and Kawai T: Innate immune peptide LL-37 displays distinct expression pattern from beta-defensins in inflamed gingival tissue. Clin Exp Immunol 146: 218-225, 2006.

30. Shahrokhi S, Ebtekar M, Alimoghaddam K, Pourfathollah AA, Kheirandish M, Ardjmand A, Shamshiri AR and Ghavamzadeh A: Substance P and calcitonin gene-related neuropeptides as novel growth factors for ex vivo expansion of cord blood CD34(+) hematopoietic stem cells. Growth Factors 28: 66-73, 2010.

31. Ding G, Liu Y, Wang W, Wei F, Liu D, Fan Z, An Y, Zhang C and Wang S: Allogeneic periodontal ligament stem cell therapy for periodontitis in swine. Stem Cells 28: 1829-1838, 2010.

32. Liu N, Shi S, Deng M, Tang L, Zhang G, Liu N, Ding B, Liu W, Liu Y, Shi $\mathrm{H}$, et al: High levels of $\beta$-catenin signaling reduce osteogenic differentiation of stem cells in inflammatory microenvironments through inhibition of the noncanonical Wnt pathway. J Bone Miner Res 26: 2082-2095, 2011. 